\title{
DEVELOPMENT OF A COMPUTER SYSTEM FOR IDENTITY AUTHENTICATION USING ARTIFICIAL NEURAL NETWORKS
}

\author{
TIMUR KARTBAEV ${ }^{\bowtie}$, BAHITZHAN AKHMETOV, AliYA DOSZHANOVA, KAIYRKHAN MUKAPIL,

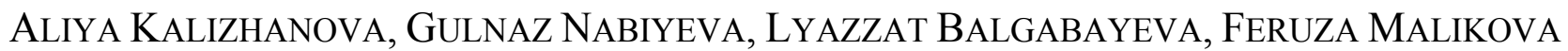 \\ ${ }^{1}$ Kazakh National Research Technical University named after K. Satpayev, Almaty, Kazakhstan \\ e-mails: Kartbayev_Timur@outlook.com; bahitzhan@rambler.ru; d_alia.81@mail.ru; kaiyrkhan@mail.ru; \\ kalizhanova_aliya@mail.ru; gulnaz_nc@mail.ru; lyazzat_iso@mail.ru; feruza-malikova@mail.ru \\ (Received August 30, 2016; revised November 22, 2016; accepted November, 2016)
}

\begin{abstract}
The aim of the study is to increase the effectiveness of automated face recognition to authenticate identity, considering features of change of the face parameters over time. The improvement of the recognition accuracy, as well as consideration of the features of temporal changes in a human face can be based on the methodology of artificial neural networks. Hybrid neural networks, combining the advantages of classical neural networks and fuzzy logic systems, allow using the network learnability along with the explanation of the findings. The structural scheme of intelligent system for identification based on artificial neural networks is proposed in this work. It realizes the principles of digital information processing and identity recognition taking into account the forecast of key characteristics' changes over time (e.g., due to aging). The structural scheme has a three-tier architecture and implements preliminary processing, recognition and identification of images obtained as a result of monitoring. On the basis of expert knowledge, the fuzzy base of products is designed. It allows assessing possible changes in key characteristics, used to authenticate identity based on the image. To take this possibility into consideration, a neuro-fuzzy network of ANFIS type was used, which implements the algorithm of Tagaki-Sugeno. The conducted experiments showed high efficiency of the developed neural network and a low value of learning errors, which allows recommending this approach for practical implementation. Application of the developed system of fuzzy production rules that allow predicting changes in individuals over time, will improve the recognition accuracy, reduce the number of authentication failures and improve the efficiency of information processing and decision-making in applications, such as authentication of bank customers, users of mobile applications, or in video monitoring systems of sensitive sites.
\end{abstract}

Keywords: artificial neural networks, facial recognition, fuzzy knowledge base, identity authentication, video monitoring system

\section{INTRODUCTION}

The systems of automatic objects recognition of various classes based on digital images are relevant to a wide range of practical solutions in the fields of computer vision (Lanitis et al., 1995), robotic engineering (Ji et al., 2012), video monitoring and access control (Rashmi et al., 2013), various interfaces of human-computer interaction (Rautaray et al., 2015). A key example reflecting the basic principles of these technologies is the automatic detection of human faces based on the electronic video picture. It is necessary to fight terrorism and crime (Iwama et al., 2013), for the general control of migration (Sanchez del Rio et al., 2016), for personal identification in bank transactions via electronic networks and a number of related tasks, where the price of mistaken identity is very high (Didimo et al., 2014; Liao et al., 2015; Fang et al.,2014).

Over the last few years, there have been proposed a lot of methods for face identification (Geman et al., 2015), on the basis of which, automatic systems for human faces recognition are developed: Smith \& Wesson (Matoso et al., 2014) (ASID system - Automated Suspect Identification System); ImageWare (Xue et al., 2015) (system FaceID); Imagis (Grother et al., 2015), Epic Solutions (Kotwal et al., 2012), Miros (Trueface system) (Sharma et al., 2013); Vissage Technology (Vissage Gallery system) (Monroe, 2009); Visionics (FaceIt system) (Parmar et al., 2016).

Artificial neural networks (NN) are widely used for tasks of pattern recognition (Jayaram et al., 2013; Vinay et al., 2013; Meruelo et al., 2016). They are used for detecting ECG signals (Gui et al., 2014), 
signature (Hatkar et al., 2015), and identification of the palm of a smartphone user (Hassanat et al., 2015). In the identity authentication systems, the mathematical apparatus of artificial NN are also widely used. For example, the recognition of justified and normalized images was performed using a simple NN. The used network calculated the face description, approximating matrix eigenvectors of the image autocorrelation (Hecht-Nielsen et al., 1989). These eigenvectors are called "eigenfaces". However, the system has not found practical application because it was based on precise justification and normalization.

The introduction of algebraic operations allows simplifying the direct computation of the "eigenfaces" (Kirby et al., 1990). For thorough coding of carefully justified and normalized images, up to 100 eigenfaces are needed. The residual error that occurs when encoding with the help of eigenfaces, is used to detect faces in unordered natural images and to determine the exact location and size of faces (Turk et al., 1991). The association of this method of faces' detection and localization with a method of eigenfaces recognition provides a reliable face recognition in real time. Thus, the environment recognition is imposed to minimal restrictions.

Models of user behavior are classified using NN with radial-basis functions (Watanabe et al., 2013). The task of identity authentication of the smartphone user is considered on the basis of the behavioral model based on the 14 gestures (Nader et al., 2015). The age of the person is recognized with the help of the fingerprints evaluation (Saxena et al., 2015). The authentication purposes based on the human face analysis are considered using a neural network approach (Khan et al., 2013). The geometric analysis of facial features is carried out using neural networks with back-propagation to determine gender of the person. Moreover, the convolutional neural networks are applied for face recognition (Sun et al., 2014).

The proposed face recognition technologies also allow automatic search and recognition in graphic files and video stream. However, to date, there are many unresolved issues in this sphere - how to teach a computer to conduct the recognition procedure according to various evaluation criteria; how to decode and store digital face images using the least amount of memory; how to select effective criteria to evaluate the similarity between faces; how to conduct a comprehensive image processing (Lin et al., 2000). The algorithms of this class must meet the following basic requirements: high recognition quality, real-time opera- tion and robustness against external factors (Hassan et al., 2015).

The recognition accuracy is $95 \%$ for frontal images (Chan et al., 2015). For images made by different vehicles and under different lighting conditions, the accuracy usually drops to $80 \%$. For images taken with a time interval of one year, recognition accuracy is approximately $50 \%$. This indicates the necessity of constant replenishment of the database with updated images and the search for more efficient algorithms.

A new artificial intelligence system called FaceNet recognizes human faces with high accuracy (Schroff et al., 2015). The result is close to $96 \%$ on a standard data set Labeled Faces in the Wild (Learned-Miller et al., 2016), which includes more than 13,000 face images taken from the Internet. A high result is provided by a new method of $\mathrm{NN}$ training: there were used triplets of pictures, which contained faces of one or different people, equally justified and taken under the same conditions. However, the problem of face recognition remains open considering aging or the impact of other changes.

Methods of NN training or self-study (Vasenkov et al., 2007) are widely used in the field of identity authentication, in particular in face recognition. Trained NN are capable of accurate reproduction of the input signal and its approximation. Automatic ability of the $\mathrm{NN}$ to interpolate allows determining the missing signals due to the influence of external factors, or the location of features, and extrapolation, in turn, may allow predicting the aging effects or changes of appearance due to a number of reasons. NN successfully restore the distorted information. They are widely used in various fields of science and technology, in particular robotic engineering and machine vision systems.

The use of methods of $\mathrm{NN}$ training or self-study may allow solving a number of related tasks in the field of person identification without the use of additional technical equipment and labor-intensive algorithms with a large number of requirements for each of the objects.

The aim of the study is to increase the effectiveness of automated face recognition to authenticate identity, considering features of face parameters change over time. To achieve these objectives, it is necessary to perform the following tasks: to consider the capabilities of intelligent biometric systems of identity authentication on the example of facial recognition, with the help of mathematical apparatus of artificial $\mathrm{NN}$; to perform the traditional approaches in the field of face 
recognition and identify their features; to propose a structure for the neural network of face recognition and to carry out simulations to verify the effectiveness of the trained $\mathrm{NN}$.

\section{METHODS AND MATERIALS}

Fig. 1 demonstrates the experimental system of observation and face recognition. IP cameras are placed across the area of the room or the street. They can be connected to a single cable, which simultaneously transfers data and power. The cameras are connected to switchboard ports DGS-1228P (PoE Switch). The $\mathrm{PoE}$ switch is also connected to the storage and monitoring server. The Internet router DIR-655 is connected to the switch for cameras' remote viewing, alerts receiving on e-mail and providing a secure access to the Internet. The presence of the network drive DNS-343 in the scheme gives you the opportunity to record from the cameras, when the storage and monitoring server is disabled, i.e., outside of working hours, the video monitoring system will become autonomous. The software D-View Cam, which supports the simultaneous work of up to 32 IP cameras, is coming with the cameras.

Multifunctional software DCS-210 D-ViewCam Standard is a comprehensive video monitoring system for corporate users, which is developed by the Taiwanese Corporation D-Link (Revadigar et al., 2015; Jang, 1993). This software provides the ability of central management and monitoring of up to 32 cameras. It is compatible with the current IP cameras and video servers of D-Link. DCS-210 provides the monitoring and recording of video, audio and events used in various applications, which provide security. The software provides a wide range of features, such as record and playback of video, and a real-time view.

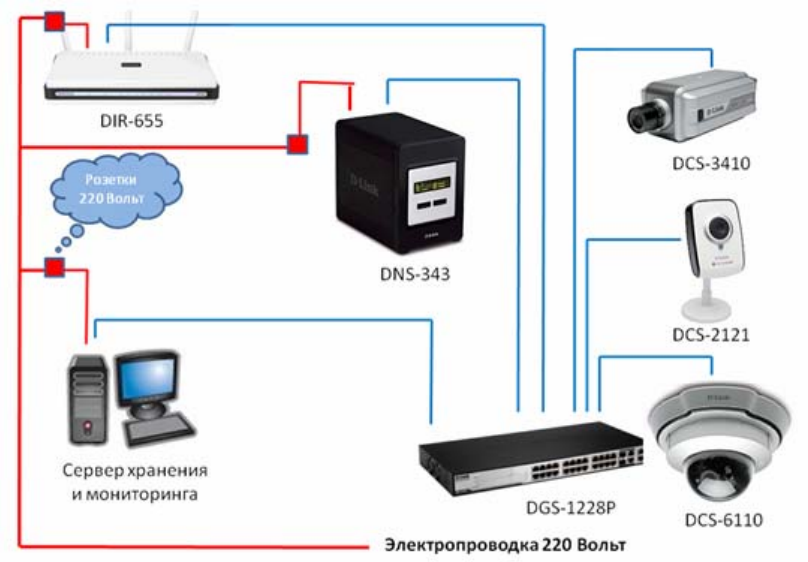

Fig. 1. Computer video monitoring system. Translation of elements: розетки 220 вольт - 220 Volt outlets; сервер хранения и мониторинга - storage and тоnitoring server; электропроводка 220 Вольт - 220 Volt power lines.

For existing computer video monitoring system, let us consider the block diagram of intelligent personality identification system (PIS) (Fig. 2), which implements the principles of processing digital information and personal recognition.

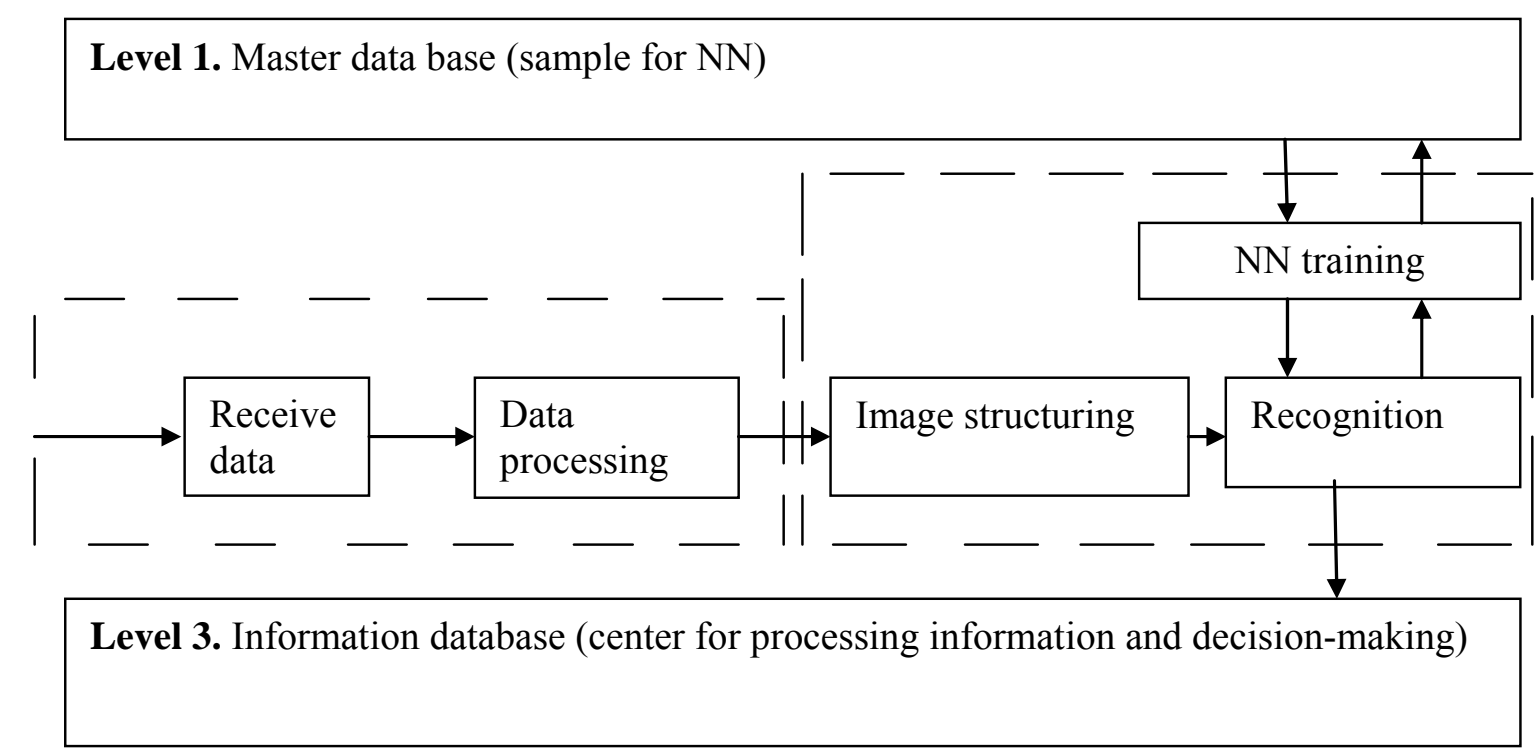

Fig. 2. Structural scheme of intellectual PIS: $a$-data processing subsystem; $b$-data distribution subsystem. 
On the upper level (1) of the system, there is a master data base comprising a set of fuzzy production rules that describe the conditions of human body aging from the expert point of view, and the speed of ageing to predict the emergence of new features on the face (wrinkles, skin pigmentation, etc.). A forecast of the expected changes is implemented by means of neuro-fuzzy networks' apparatus of the ANFIS type (Jang, 1993). This type of network uses the Sugeno algorithm of fuzzy inference. Moreover, it is an effecttive tool because of the low value of learning error, consideration of expert opinion and extrapolation of output values. The level 1 also includes the database (DB) of references in the form of a set of points with coordinates of people features or functions describing the location of features relative to each other (eyes, nose, mouth, chin, etc.). The NN training is held with the help of reference samples in the database.

The second level (2) includes the adjustment of the NN weighting factors, assessment of training effecttiveness, possible correction of distorted image signals, the forecast of personal features' changes and the reporting on person.

The third level (3) contains the information database (IDB). Data on the identified personalities are transferred to the IDB.

\section{RESULTS}

\section{RECOGNITION OF THE PRESENTED IMAGE ACCORDING TO GRAPHIC COORDINATES (PIXELS)}

The feature of image analysis in the system of computer video monitoring is that the obtained images may be distorted, i.e., contain artifacts. This must be considered in their processing. In addition, the master database (Fig. 2) contains samples of face images, which are compared in the process of identity authentication. Over time, a person undergoes some changes, including wrinkles, change of the contours, skin pigmentation, etc. Such changes cannot be accounted in the master database, therefore the method of image processing should take into account the possibility of temporal changes on the human face and evaluate the image, taking into account forecasts of changes of some parameters over time.

In this regard, this work uses a hybrid NN, which combines the benefits of fast learning of artificial NN and possible fuzzy inference based on expert infor- mation that is a key feature of the developed computer system. This approach allows taking into account the forecast of age-related changes in facial features and using it to compare images obtained from video monitoring systems to standard samples.

A new image (photograph) enters the input of PIS (Fig. 3a). At the initial stage, the pre-processing of the original image takes place. The original color image is converted to grayscale (Fig. 3b) using commands in MatLab (Porshnev, 2010) in order to simplify further calculations and the software implementation. Further, to improve the contrast of an image, the pixel values of the original image (Table 1) are converted so that the histogram of pixels' brightness of the obtained image corresponds to the desired histogram (Fig. 3c). At the next stage, the grid imposition of coordinate points takes place. This highlights key signs or characteristics of the face (Fig. 3d).

Table 1. A program fragment.

RGB=imread ('brad.jpg');

$>>$ I=rgb2gray (RGB);

$>>\mathrm{J}=$ histeq $(\mathrm{I})$;

$>$ Fig.;

$>$ subplot $(2,2,1)$;imshow (I);

$>>$ subplot $(2,2,2)$;imhist (I);

$>>$ subplot $(2,2,3)$;imshow $(\mathrm{J})$;

>> subplot (2,2,4);imhist (J);

Key facial features are proportional and can be described by linear functions. We use linear $\mathrm{NN}$ with one input and one output As a learning example (remembering the coordinates of five points $\left.Y=\left[\begin{array}{lllll}20 & 40 & 60 & 80 & 90\end{array}\right] ; X=\left[\begin{array}{lllll}80 & 60 & 50 & 48 & 45\end{array}\right]\right)$.

Fragment of the program for NN development and testing is shown in the package Neuro Toolbox (MatLab) in Fig. 4. The network is trained according to the algorithm of the inverse error propagation (Ivanov, 2004). As can be seen from the values of Fig. 4, NN correctly identifies the Y coordinate point.

Experiment on the superposition of interference and restoring the distorted information (points restoring)

The initial values of $\mathrm{X}, \mathrm{Y}$ are encoded with the purpose of saving data from the external influence caused by restrictions, speckle, luminance, weather effects, etc. Therefore, knowing the exact coordinates of a point is confirmed by the value of 1 , and distorted -1. (Table 2) 


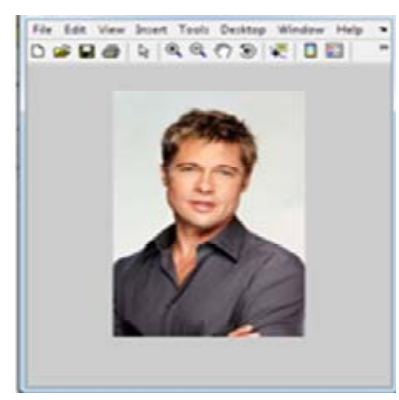

a - color picture

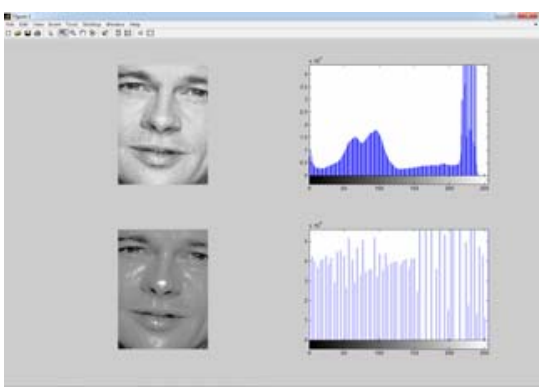

c - enhancement of contrast and histogram of the image

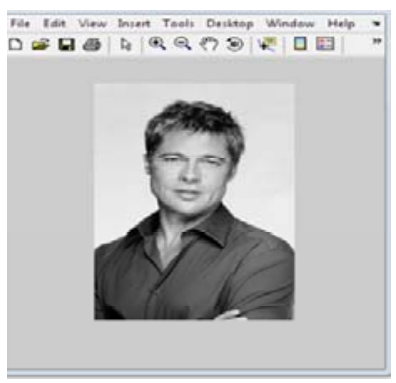

b - grayscale image

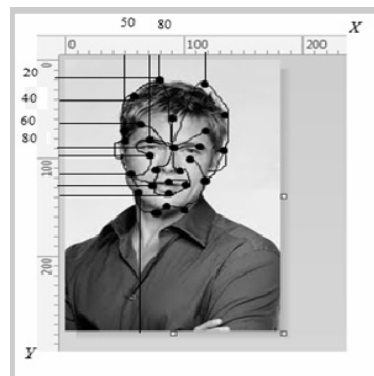

d - Part of coding image system work for NN (coordinate grid imposition)

Fig. 3. The analyzed image.

1. untitled/Neural Network *

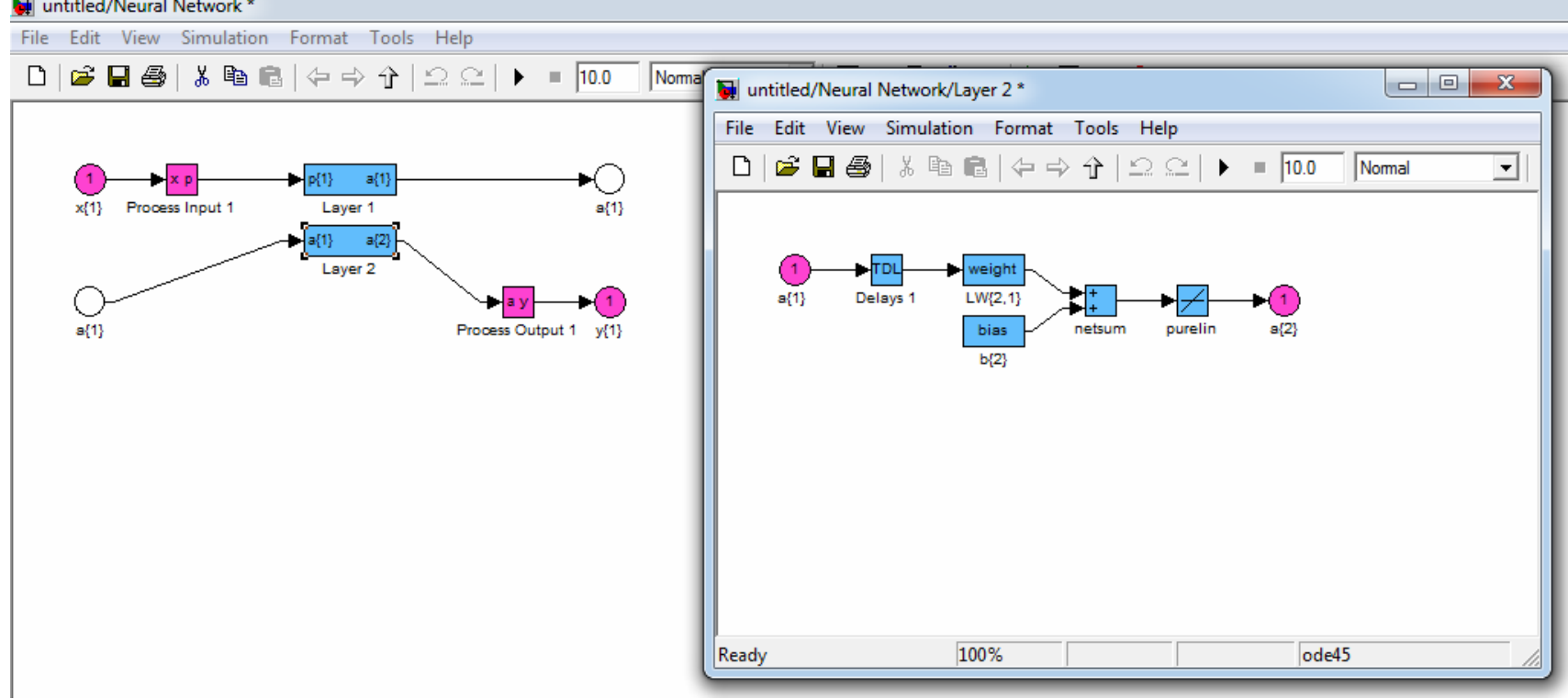

Fig 4. NN structure.

Table 2. The coded coordinates.

\begin{tabular}{|c|c|}
\hline & $Y_{1}$ \\
\hline$X_{1}$ & 1 \\
\hline$X_{2}$ & 1 \\
\hline$X_{3}$ & 1 \\
\hline$X_{4}$ & 1 \\
\hline$X_{5}$ & 1 \\
\hline
\end{tabular}


Intelligent system imposes a new coordinate system corresponding to the output of the bipolar threshold (Signum) NN activation function (Fig. 5).

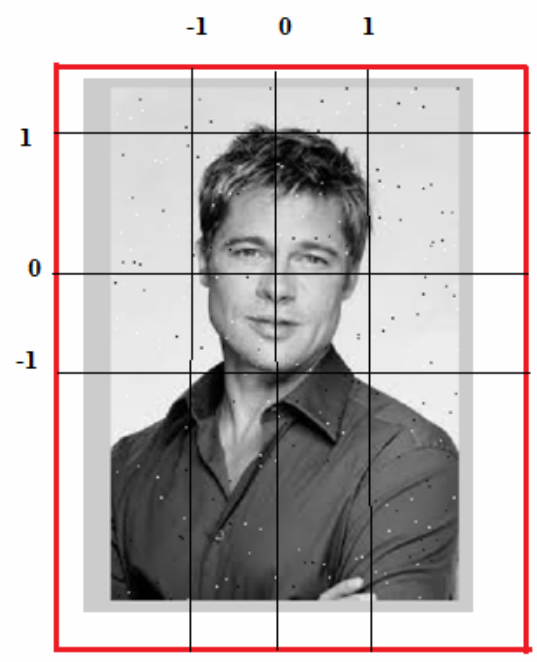

Fig. 5. Threshold coordinate system.

Let us consider a Hopfield network (Hopfield, 1982). In the Hopfield network, the input signals of neurons are also the output signals of the network: $\mathrm{x}_{\mathrm{i}}(\mathrm{k})=\mathrm{y}_{\mathrm{i}}(\mathrm{k}-1)$, while stimulating vector is not particularly important. In the classical Hopfield system, there is no connection of the neuron with its own output, which corresponds to $w_{i i}=0$, and the whole weight matrix is symmetric: $\mathrm{w}_{\mathrm{ij}}=\mathrm{w}_{\mathrm{ji}}$.

$$
\hat{W}=\hat{W}^{T} .
$$

The symmetry of the weight matrix guarantees the convergence of the learning process. The learning process of network creates the zone of attraction of some equilibrium points, corresponding to the training data. When using associative memory, we are dealing with the training vector $\mathbf{x}=\left(x_{1}, x_{2}, \ldots x_{n}\right)$ or set of those vectors that identify the location of specific points of attraction (attractors) as a result of learning.

Each neuron has an activation function Signum with values \pm 1 :

$$
\operatorname{sign}(a)=\left\{\begin{array}{c}
1, a \geq 0 \\
-1, a<0
\end{array} .\right.
$$

This means that the output signal of the i-th neuron is determined by the function:

$$
y_{i}=\operatorname{sign}\left(\sum_{j=1}^{N} w_{i j} x_{j}+b_{i}\right),
$$

where $\mathrm{N}$ is the neuron number, $\mathrm{N}=\mathrm{n}$. Often the constant component $b_{i}$, which determines the threshold of individual neurons, equals to $0, \mathrm{a}$ - the output signal.

To check the work effectiveness of trained NN to the possibility of recovery of the distorted signal, let us perform calculations.

Let the encoded signals input the NN; they represent the coordinates of the facial features:

$$
\begin{gathered}
x_{1}^{T}=\left(\begin{array}{lllll}
-1 & 1 & -1 & -1 & -1
\end{array}\right) \\
x_{2}^{T}=\left(\begin{array}{lllll}
1 & -1 & 1 & -1 & 1
\end{array}\right) \\
x_{3}^{T}=\left(\begin{array}{lllll}
-1 & 1 & -1 & -1 & -1
\end{array}\right) \\
x_{4}^{T}=\left(\begin{array}{lllll}
-1 & 1 & -1 & -1 & 1
\end{array}\right) \\
x_{5}^{T}=\left(\begin{array}{lllll}
-1 & 1 & -1 & 1 & -1
\end{array}\right)
\end{gathered}
$$

where $x_{i}$ - the current value of the signal.

The weight coefficients of the NN matrix for images are determined by the formula:

$$
W_{i}=x_{i} x_{i}^{T},
$$




$$
\begin{aligned}
& W_{1}=x_{1} x_{1}^{T}=\left(\begin{array}{rrrrr}
1 & -1 & 1 & 1 & 1 \\
-1 & 1 & -1 & -1 & -1 \\
1 & -1 & 1 & 1 & 1 \\
1 & -1 & 1 & 1 & 1 \\
1 & -1 & 1 & 1 & 1
\end{array}\right) \text {, } \\
& W_{2}=x_{2} x_{2}^{T}=\left(\begin{array}{rrrrr}
1 & -1 & 1 & -1 & 1 \\
-1 & 1 & -1 & 1 & -1 \\
1 & -1 & 1 & -1 & 1 \\
-1 & 1 & -1 & 1 & -1 \\
1 & -1 & 1 & -1 & 1
\end{array}\right) \text {, } \\
& W_{3}=x_{3} x_{3}^{T}=\left(\begin{array}{rrrrr}
1 & -1 & 1 & 1 & 1 \\
-1 & 1 & -1 & -1 & -1 \\
1 & -1 & 1 & 1 & 1 \\
1 & -1 & 1 & 1 & 1 \\
1 & -1 & 1 & 1 & 1
\end{array}\right) \text {, } \\
& W_{4}=x_{4} x_{4}^{T}=\left(\begin{array}{rrrrr}
1 & -1 & 1 & 1 & -1 \\
-1 & 1 & -1 & -1 & 1 \\
1 & -1 & 1 & 1 & -1 \\
1 & -1 & 1 & 1 & -1 \\
-1 & 1 & -1 & -1 & 1
\end{array}\right) \text {, } \\
& W_{5}=x_{5} x_{5}^{T}=\left(\begin{array}{rrrrr}
1 & -1 & 1 & -1 & 1 \\
-1 & 1 & -1 & 1 & -1 \\
1 & -1 & 1 & -1 & 1 \\
-1 & 1 & -1 & 1 & -1 \\
1 & -1 & 1 & -1 & 1
\end{array}\right) \text {. }
\end{aligned}
$$

The resulting weight matrix of the network $\mathrm{W}$ can be calculated using the formula:

$$
W=\sum_{i=1}^{5} W_{i}=\left(\begin{array}{rrrrr}
5 & -5 & 5 & 1 & 3 \\
-5 & 5 & -5 & -1 & -3 \\
5 & -5 & 5 & 1 & 3 \\
1 & -1 & 1 & 5 & -1 \\
3 & -3 & 3 & -1 & 5
\end{array}\right)
$$

To determine the reference image stored in memory, we need to calculate the product $W X_{i}=Y_{i}$ $(i=1,2,3,4,5)$. For example, when applying $x_{3}$, we have:

$$
W x_{3}=\left(\begin{array}{rrrrr}
5 & -5 & 5 & 1 & 3 \\
-5 & 5 & -5 & -1 & -3 \\
5 & -5 & 5 & 1 & 3 \\
1 & -1 & 1 & 5 & -1 \\
3 & -3 & 3 & -1 & 5
\end{array}\right) \times\left(\begin{array}{r}
-1 \\
1 \\
-1 \\
-1 \\
-1
\end{array}\right)=\left(\begin{array}{r}
-19 \\
19 \\
-19 \\
-7 \\
-13
\end{array}\right) .
$$

Taking into account a threshold activation function (2), we get the output signal $\left(\begin{array}{lllll}-1 & 1 & -1 & -1 & -1\end{array}\right)$, that is the neural network have correctly restored the saved image $x_{3}$. Similar results were obtained for the other images.

Let the image $x_{3}$, distorted in the second and fourth points, input the network. The image simulates a decrease of signal quality.

$$
\bar{x}_{3}{ }^{T}=(-1-1-1 \quad 1-1)
$$

Using the matrix $\mathrm{W}_{\kappa} \bar{x}_{3}^{T}$, we get:

$$
W_{x_{3}}=\left(\begin{array}{ccccc}
5 & -5 & 5 & 1 & 3 \\
-5 & 5 & -5 & -1 & -3 \\
5 & -5 & 5 & 1 & 3 \\
1 & -1 & 1 & 5 & -1 \\
3 & -3 & 3 & -1 & 5
\end{array}\right) \times\left(\begin{array}{c}
-1 \\
-1 \\
-1 \\
1 \\
-1
\end{array}\right)=\left(\begin{array}{c}
-7 \\
7 \\
-7 \\
5 \\
9
\end{array}\right)
$$

Taking into account the activation function, we get $\mathrm{x}_{3}=\left(\begin{array}{lllll}-1 & 1 & -1 & 1 & -1\end{array}\right)$, that is NN fixed the second coordinate, but could not correct the fourth, therefore, NN needs further study.

It is known that most systems of face recognition do not have the ability to forecast changes of key facial features over time, i.e., aging. To consider this possibility, the author have proposed a neuro-fuzzy network (NFN) of an ANFIS type (Jang, 1993), which implements the Tagaki-Sugeno algorithm (Takagi, 1985). To construct the NFN, we used the editor "anfis edit" from the MatLab package. The NFN is based on a fuzzy inference system that implements the opinions and experiences of experts in the field of human aging.

\section{EXPERIMENT ON NEURO-FUZZY PREDICTION OF THE EXTENT OF HUMAN AGING, I.E., CHANGE OF THE FACE KEY FEATURES}

The expert determines the dependence of the degree of human body aging (face parameters), depending on a number of factors, such as health, nutrition, stress, heredity, environment, degree of self-realization. 
Fuzzy knowledge base consists of 15 rules of the type IF "Condition .." THEN "Conclusion", for example, one of the rules is as follows:

IF health is good AND nutrition is good AND resistance to stress is high AND the environment is good AND the self-realization is high, THEN the levels of aging are low, etc.
General view of the fuzzy inference system is shown in Fig. 6. Expert chooses the type and number of membership functions (Fig. 7).

After the implementation of the knowledge base, we have checked the operation of fuzzy expert system (fuzzy inference system) Fig. 8a. A three-dimensional image of an interaction between the degree of aging and nutrition and health is shown in Fig. 8b.

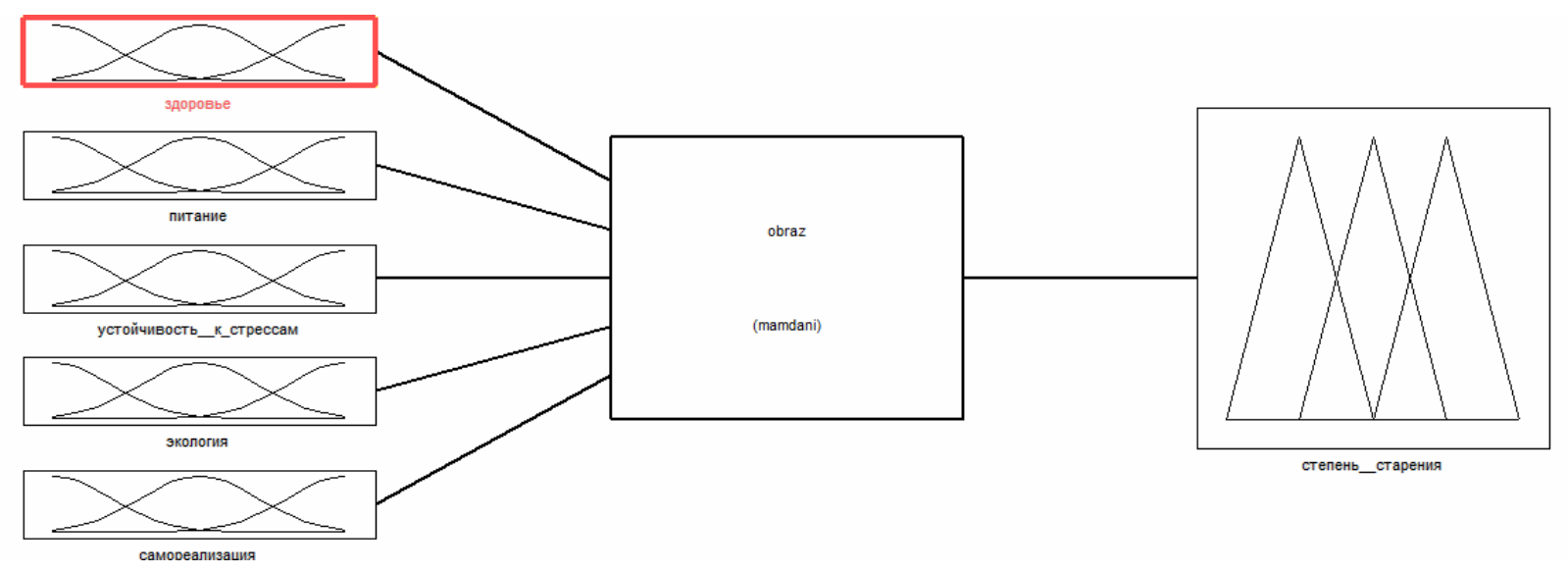

Fig. 6. General view of the fuzzy inference system. Translation of elements: здоровье - health; питание nutrition; устойчивость к стрессам - resistance to stress; экология - environment; самореализация - selfrealization; obraz - image; степень старения - level of aging.

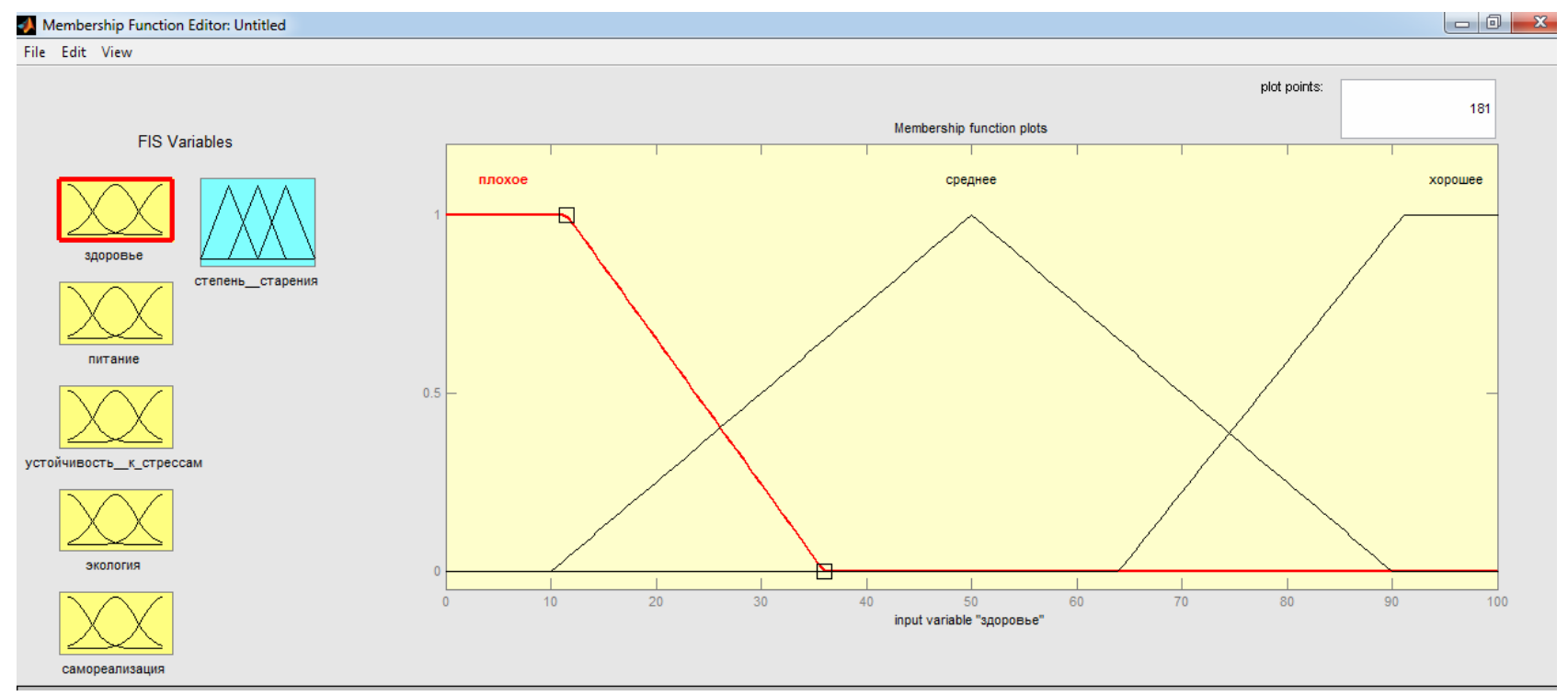

Fig. 7. The membership functions of the variable "health". Translation of elements: здоровье - health; питание nutrition; устойчивость к стрессам - resistance to stress; экология - environment; самореализация - selfrealization; плохое - bad; среднее - average; хоромее - good. 


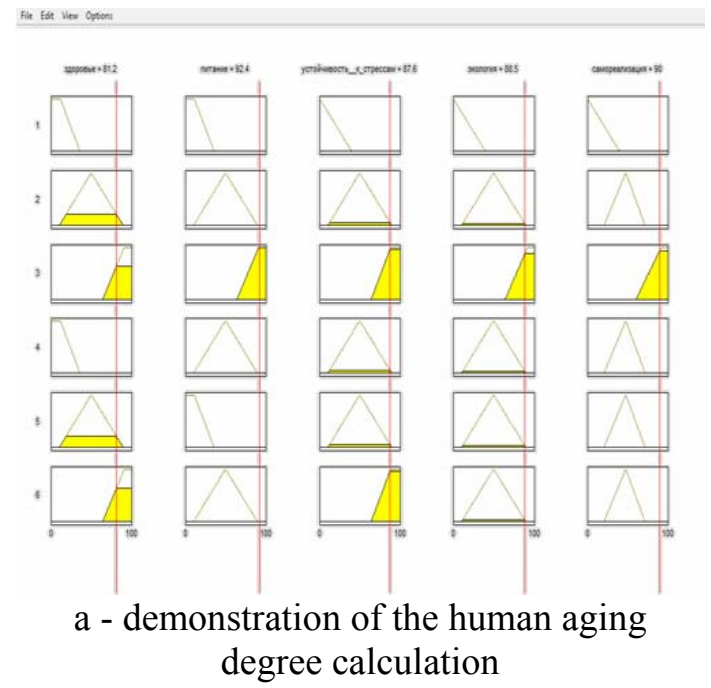

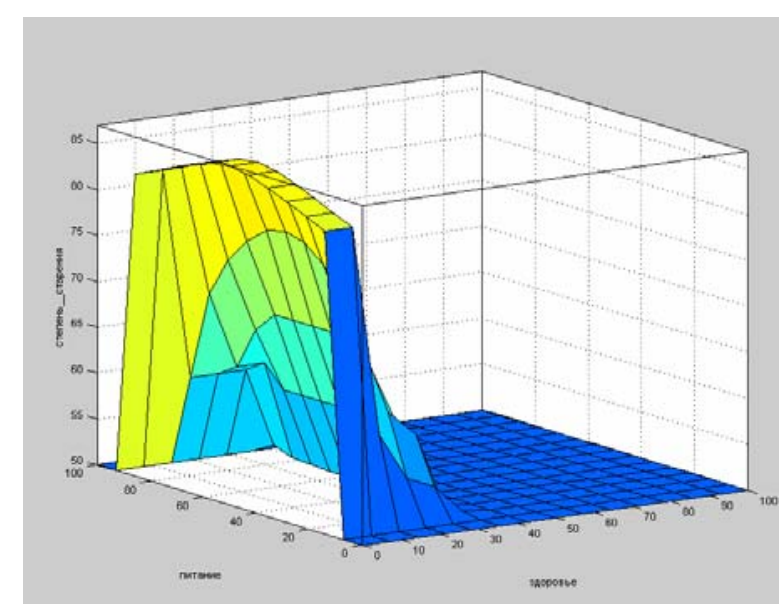

$\mathrm{b}$ - representation of aging factors

Fig. 8. The test of the fuzzy expert system (fuzzy inference system) operation. Translation of elements: здоровье - health; питание - nutrition; устойчивость к стрессам - resistance to stress; экология - environment; самореализаиия - self-realization; степень старения - level of aging.

Based on the numerical values of the aging factors from the resulting fuzzy inference system, we have defined the test sample (Fig. 9) for training NFN with the aim of obtaining forecast values.

In the program "anfis edit", the NFN is implemented (Fig. 10A). It has 5 inputs and 1 output, corresponding to a fuzzy system. The network is trained based on the gradient method. The number of learning cycles is equal to 30 (Fig. 10b).

To check the work accuracy of NFN, the forms and values of three-dimensional images of aging factors in the fuzzy system (Fig. 4) and NFN (Fig. 10c) were compared. We can see that key values are identical.

To check the effectiveness of NFN work, we determined the predictive value of the aging degree, i.e., the emergence of new features on the face (Table 3). The value of 5.73 means that at the high estimates of the input factors, the speed of biological processes in facial features is slow, i.e., for the presented object (face photos of actor Brad Pitt) of the PIS neural network, the reference model may be stored in the database for a long time without essential changes of coordinates.

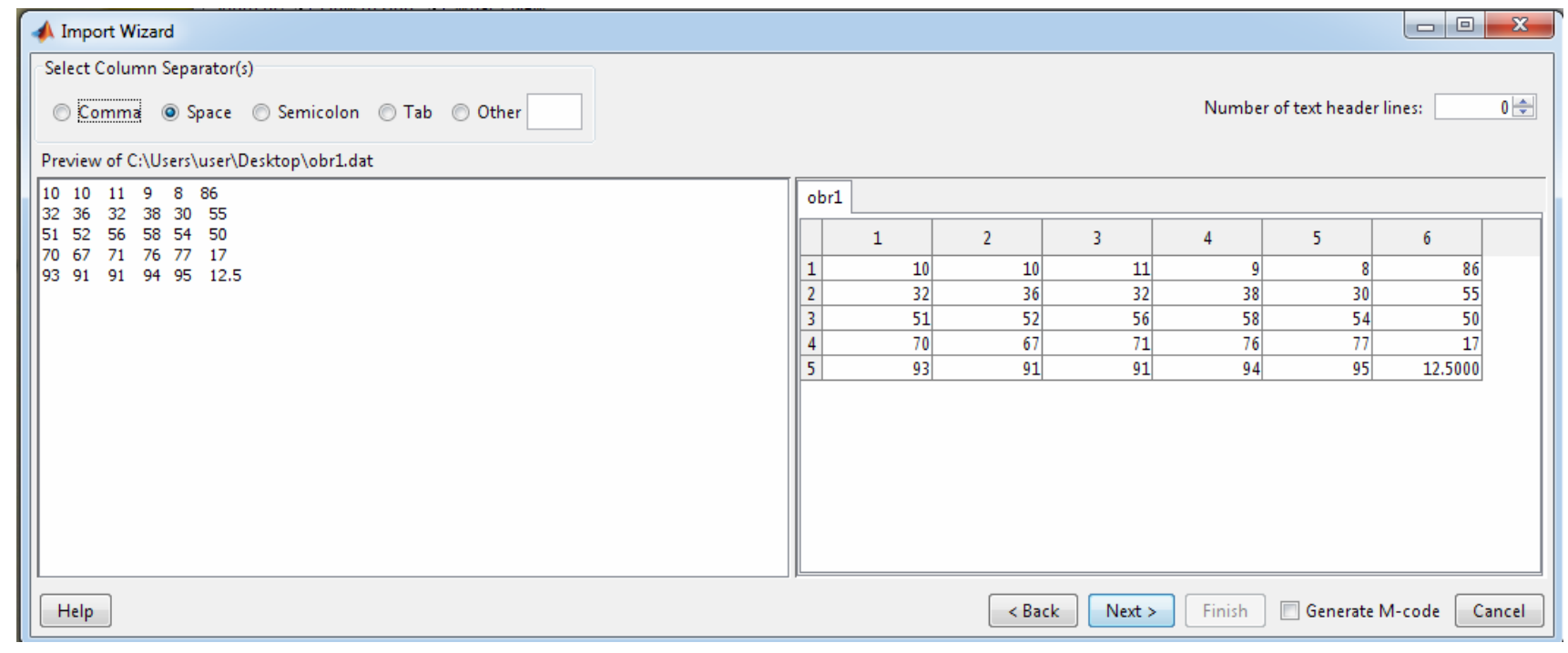

Fig. 9. Establishment of test samples for NFN. 


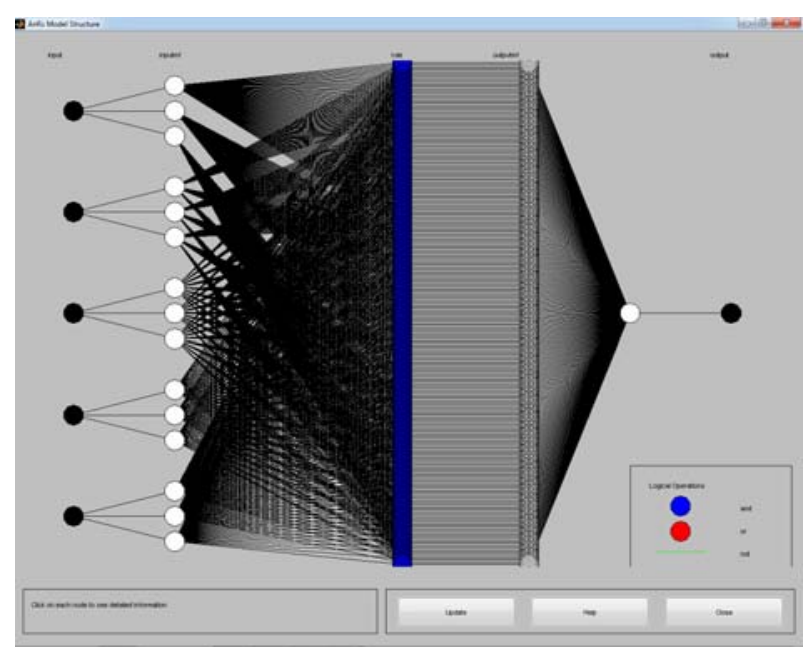

a - General view of the NFN of aging function

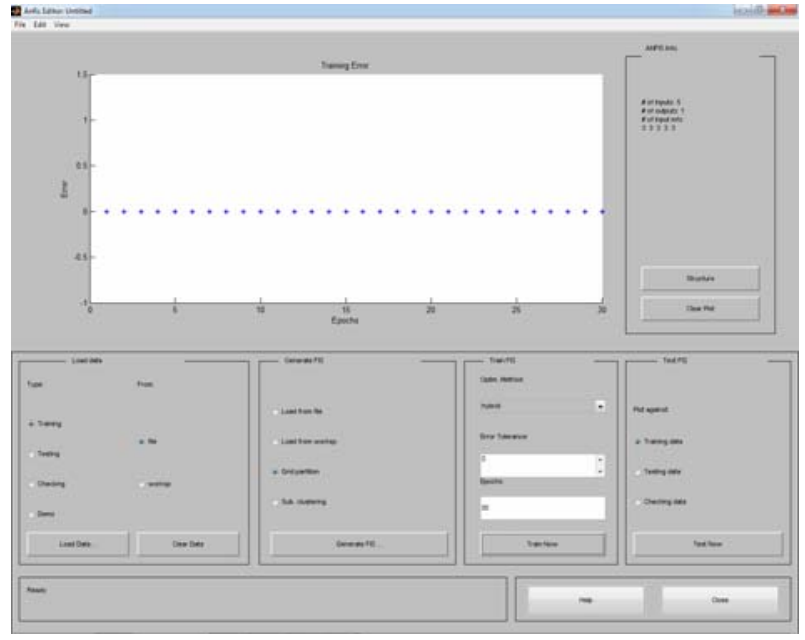

b - Software training of NFN

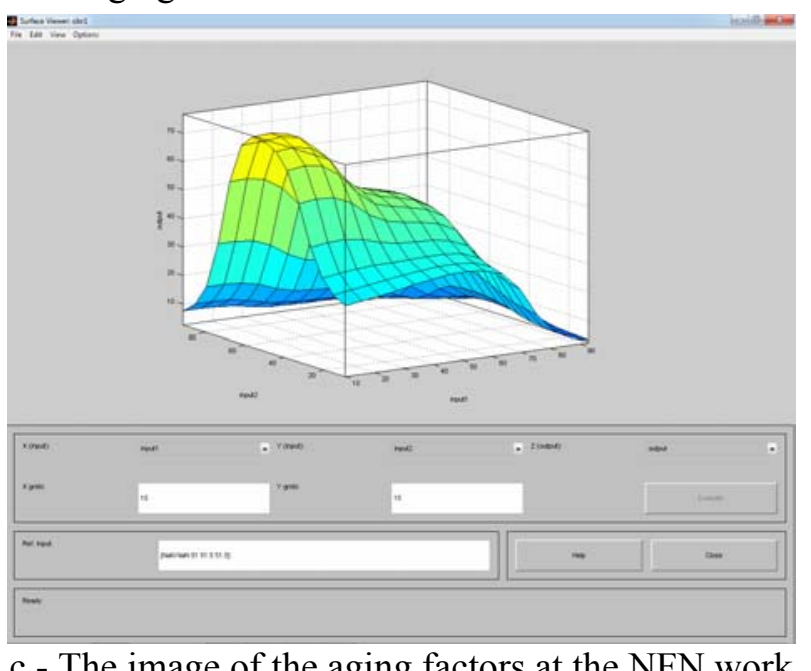

Fig. 10. NFN.

Table 3. Program listing for definition of estimated values.

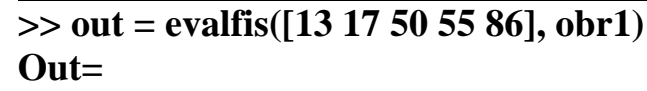

\section{DISCUSSION}

Application of neural systems in computer system of video monitoring and authentication opens up broad prospects for the analysis and processing of obtained images. Despite the good experimental results, the proposed approach has several limitations. First of all, during the preparation of the problem solution, it is not always possible to determine the exact amount of input data in the training, which significantly affects learning outcomes and, as a consequence, the results of the network operation. Second, when solving real problems using neural networks, the challenge is to link output indicators with the available data. That is why we often use an excessive amount of data. Third, an attempt to consider more options, including the formation of fuzzy rules of productions, increases the dimensionality of the network, increases the learning time and reduces the quality of network operation.

It should be noted that to implement the proposed approach, the neurocomputers developed by Adaptive Solutions (USA) (Razi et al., 2015) or Hitachi (Japan) (Lydia-Marie Joubert, 2016) could be used. Hitachi neurocomputers allow you to implement NN, including up to 576 neurons. In addition, the computers of Mark family produced by TRW (Sinitsyna, 2015) are well-known representatives of these systems. Mark IV is a uniprocessor with pipelined architecture. It maintains up to 236 thousand virtual processing elements, which allows handling up to 5 million interconnects/s. Computers of Mark family have a common software shell ANSE, providing software compatibility of models with $\mathrm{NN}$ emulator (Meruelo et al., 2016). 
There are many methods for the face identifycation that can be divided into several categories:

1. Methods based on the knowledge (Grother et al., 2015). They use expert knowledge about the shape and position of facial features, their geometric proportions.

2. Methods based on invariant properties (Parmar et al., 2016). They are looking for invariant properties, which are peculiar for a face under changing illumination conditions, facial expressions, its position relative to the camera. In the first stage, the candidates are defined according to the main global properties, such as, skin color, face size and shape. The system then checks the selected candidates with the help of local properties eyebrows, eyes, nose, mouth and hair.

3. Group of methods based on comparison with the sample (Schroff et al., 2015). In these methods, the system selects several face samples or samples of certain face features and calculates the correlation between the input image (picture) and reference image within the database. Graphic location of facial features is specified, usually in the form of parameters of some functions. Next, the image (a human face) is recognized with the help of the value of the correlation coefficient. Based on the methods of the third group, the program OpenCV (Open Computer Vision) operates (Andersen et al., 2015). It is a computer vision library with an open source code, which provides a set of data types and numerical algorithms to process the image with the help of computer vision. The EigenFaces algorithm is used to train the model (Diniz et al., 2015). The algorithm is based on the use of statistical characteristics, such as expectation function and covariance matrice. Recognition quality depends on the chosen method, and on the number and quality of the images used in the calculations, that is a disadvantage of the package.

4. Methods based on learning (Vasenkov et al., 2007). The algorithms, based on these methods, use mathematical models, which are trained using a set of reference or test images. For example, the learning algorithms that are implemented in neural networks. Then trained $\mathrm{NN}$ are used in identification systems.

It should be noted that the first three areas have a number of disadvantages. In the preparation of detailed laws or conditions, the recognition system will reject the faces, which do not fully meet the conditions. If the laws are generalized, it can lead to a large number of incorrect detections. It is also difficult to develop rules to detect faces with different facial expressions and in different projections, it is necessary to foresee all possible cases that is a time-consuming task.

The problem of the second group methods is that when searching for a permanent facial features and transferring images to digital form, these characteristic can be distorted by speckle and light levels. The face may have a little noticeable border, while shadows can give a sharp edge that will lead to incorrect operation of the algorithm. This requires additional hardware and software that implement the methods of filtering and restoration of distorted information.

Methods based on comparison of the submitted picture with the standard, or sample show a low level of confidence, as they are not always able to deal with a variety of shapes, positions, sizes. Moreover, they do not take into account the appearance of glasses or hearing aids, hats, facial hair, etc., and the factor of human aging, i.e., modification of the submitted characteristics in time.

As a result of the study, a method of personal recognition and identification is developed, taking into account possible changes of key facial features over time. The proposed approach is based on the results of the existing models analysis and methods of face recognition.

The basic idea of the proposed approach is that the authentication process is split into several stages: first is the image pre-processing (the image color is converted to grayscale, the contrast is improved); second, the key characteristics are selected based on image analysis and the received characteristics are coded; third, the recognition of the image taking into account the changes forecast of key parameters. In addition, fuzzy rules of productions are developed, on the basis of which the problem of forecasting changes in key parameters is solved.

Based on the analysis of hybrid fuzzy-network systems, we can state that it is advisable to use the adaptive system neuro-fuzzy output - ANFIS in order to build a system of evaluation and identification of obtained image. In the ANFIS system, the conclusions are based on fuzzy logic system, and the parameters of the membership function are adjusted using the error back-propagation algorithm. This approach helps 
to identify patterns and discover new correspondences, to predict the values of key parameters of authentication. Comparison of the training results shows good consistency with the experimental data obtained by evaluating characteristics. The solution may serve as a basis for the hardware implementation of the proposed neuro-fuzzy algorithm when solving the problems of identity authentication in terms of hardware and software complex of video monitoring and face recognition.

Analysis of the conducted experiments shows that the application of the ANFIS system for the personal authentication is very promising. The disadvantage is that the quality of the results significantly depends on the quality of experimental data and on the volume of training samples. Therefore, the selection of training samples is an important issue when using ANFIS. Studies have shown that the algorithm of Sugeno-type gives high performance, but involves quite complex learning procedure. The obtained results correspond to the conclusions of some other researchers who studied the possibility of applying the neural network approach to the solution of various problems (Matoso et al., 2014; Xue et al., 2015; Jang, 1993). The accuracy of obtained numerical estimates is provided by using standard means of MATLAB.

Today, there are many different situations, in which well-known algorithms for face recognition cease to work effectively and allow a large percentage of errors. A new generation of recognition systems must be able to identify a person in real-time and in environments with much fewer restrictions. To reach this purpose, the identification systems should work effectively in the natural environment - in the presence of speckle and variable illumination. They cannot be based on the use of one identification category - a very important feature is the analysis according to different categories. With this purpose, the author proposed an approach based on $\mathrm{NN}$, which is able to solve the set tasks. If we talk about wearable systems, their sensing elements require small size, low power consumption and the ability to fix on clothes. Considering these requirements, we can assume that the system with the means of face recognition, which implements the algorithms of trained $\mathrm{NN}$, have the greatest potential for wide application.

Nevertheless, to be widely applicated, the facial recognition systems should learn to interact with the user without creating discomfort. This means that future intelligent systems must use the same recognition categories as people and have the ability to imitate human thinking. Further research will continue in this direction, but it is already clear that the objectives are achievable.

\section{CONCLUSIONS}

As a result of conducted study, we can draw the following conclusions.

1. The approach for the implementation of computer authentication system is proposed, based on artificial neural networks, which allows not only comparing the obtained image with the reference samples, which are stored in the database, but also taking into account the forecast changes in key characteristics over time (e.g., due to aging).

2. Structural diagram of a hardware-software complex for identity authentication is developed. It has a three-tier architecture and implements preliminary processing, recognition and identification of images obtained as a result of monitoring.

3. On the basis of expert knowledge, the fuzzy base of products is designed. It allows assessing possible changes in key characteristics, used to authenticate identity based on the image. To take this possibility into consideration, a neuro-fuzzy network of ANFIS type was used, which implements the algorithm of Tagaki-Sugeno.

4. The conducted experiments, implemented with the help of MatLab, showed high efficiency of the developed neural network and a low level of learning errors, which allows us to recommend this approach for practical implementation.

The significance of the results for practical use is determined primarily by the developed system of fuzzy productions that allow predicting face changes over time.

\section{REFERENCES}

Andersen MR, Jensen T, Lisouski P, Mortensen AK, Hansen MK, Gregersen T, et al. (2015). Kinect Depth Sensor Evaluation for Computer Vision Applications. Tech Rep Electron Comput Eng 1:1-35.

Chan TH, Jia K, Gao S, Lu J, Zeng Z, Ma Y (2015). PCANet: A Simple Deep Learning Baseline for Image Classification? IEEE Trans Image Process 24:5017-32.

Didimo W, Liotta G, Montecchiani F (2014). Network visualization for financial crime detection. J Vis Lang Comput 25:433-51.

Diniz FA, Reis Da Silva T, Eduardo F, Alencar S (2015). An Empirical Study Of the Behavior of a Face Recognition System Based on Eigenfaces and K- Nearest Neighbors Techniques. Int Conf Eng. Comput Educ 9:22-6. 
Fang H, Mac Parthaláin N, Aubrey AJ, Tam GKL, Borgo $\mathrm{R}$, Rosin PL, et al. (2014). Facial expression recognition in dynamic sequences: An integrated approach. Pattern Recognit 47:1271-81.

Geman D, Geman S, Hallonquist N, Younes L (2015). Visual Turing test for computer vision systems. Proc Natl Acad Sci 112:3618-23.

Grother P, Shevtsov D, Tabassi E, Wolf A (2015). Face Recognition Standards. Encycl Biometrics 1:467-75.

Gui Q, Jin Z, Xu W (2014). Exploring EEG-based biometrics for user identification and authentication. Signal Process Med Biol 1:1-6.

Hassan MY, Khalifa OO, Talib AA, Abdulla AH (2015). Unconstrained Facial Recognition Systems. A Review, Asian J Appl Sci 3:346-54.

Hassanat A, Al-Awadi M, Btoush E, Al-Btoush A (2015). New mobile phone and webcam hand images databases for personal authentication and identification. Procedia Manuf 3:4060-7.

Hatkar P, Salokhe B, Malgave A (2015). Offline Handwritten Signature Verification using Neural Network. Methodology 2:1-5.

Hecht-Nielsen R (1989). Self-Organization and Associative Memory. IEEE J. Quant Electron 25:237.

Hopfield J (1982). Neural networks and physical systems with emergent collective computational abilities. Proc Natl Acad 79:2554-8.

Iwama H, Muramatsu D, Makihara Y (2013). Gait verification system for criminal investigation. IPSJ Trans Comput Vis Appl 5:163-75.

Jang J (1993). ANFIS: adaptive-network-based fuzzy inference system. IEEE Trans. Syst. Man 23:665-85.

Jayaram M, Fleyeh H (2013). Soft Computing in Biometrics: A Pragmatic Appraisal. Am J Intell Syst 3:105-12.

Ji W, Zhao D, Cheng F, Xu B, Zhang Y, Wang J (2012). Automatic recognition vision system guided for apple harvesting robot. Comput Electr Eng 38:1186-95.

Khan A, Gour B (2013). Gender Classification Technique Based on Facial Features using Neural Network. Int J Comput Sci Inf Technol 4:839-43.

Kirby M, Sirovich L (1990). Application of the KarhunenLoeve procedure for the characterization of human faces. IEEE Trans Pattern Anal 12:103-8.

Kotwal N, Li J, Sandy J, Plaas A, Sumner DR, Mow VC, et al. (2012). Initial application of EPIC- $\mu C T$ to assess mouse articular cartilage morphology and composition: effects of aging and treadmill running. Osteoarthritis Cartilage 20:887-95.

Lanitis A, Taylor CJ, Cootes TF (1995). An Automatic Face Identification System Using Flexible Appearance Models. Image Vis Comput 13:393-401.

Learned-Miller E, Huang GB, RoyChowdhury A, Li H, Hua G (2016). Labeled Faces in the Wild: A Survey. Adv. Face Detect. Facial Image Anal 1:189-248.
Liao S, Hu Y, Zhu X, Li SZ (2015). Person Re-Identification by Local Maximal Occurrence Representation and Metric Learning, Proc. IEEE Conf. Comput. Vis. Pattern Recognit 1:2197-206.

Lin SH (2000). An Introduction to Face Recognition Technology (PDF) - Semantic Scholar, Informing Sci. Spec. Issue Multimed. Informing Technol 3:1-7.

Lydia-Marie J, McDonald K (2016). SEM Visualization of Biological Samples using Hitachi Ionic Liquid HILEM® IL 1000: a Comparative Study. Microsc Microanal 22: 1170-1.

Matoso RI, Freire AR, Santos LSM, Daruge EJ, Rossi AC, Prado FB, et al. (2014). Comparison of Gunshot Entrance Morphologies Caused by .40-Caliber Smith \& amp; Wesson, .380-Caliber, and 9-mm Luger Bullets: A Finite Element Analysis Study. PLoS One 9:111-92

Meruelo A, Simpson D, Veres S, Newland P (2016). Improved system identification using artificial neural networks and analysis of individual differences in responses of an identified neuron. Neural Networks 75:56-65.

Monroe DA (2009). Method for incorporating facial recognition technology in a multimedia video monitoring system US7634662 B2.

Nader J, Alsadoon A, Prasad P, Singh A (2015). Designing Touch-Based Hybrid Authentication Method for Smartphones. Procedia Comput 70:198-204.

Parmar DN, Mehta BB (2016). Face Recognition Methods \& Applications. Int.J.Computer Technol. Appl 4:84-6.

Porshnev S (2010). MATLAB 7. Fundamentals of work and programming - Everything for student. Moscow: Binom-Press $151 \mathrm{p}$.

Rashmi R, Latha B (2013). Video monitoring system and facility to access Pc from remote areas using smart phone. Int. Conf. Inf. Commun. Embed. Syst. IEEE 2013:491-5.

Rautaray SS, Agrawal A (2015). Vision based hand gesture recognition for human computer interaction: a survey. Artif Intell Rev 43:1-54.

Razi M, Attar PJ, Vedula P (2015). Uncertainty quantification of multidimensional dynamical systems based on adaptive numerical solutions of the Liouville equation. Probabilistic Eng Mech 42:7-20.

Revadigar G, Javali C, Hu W, Jha S (2015). DLINK: Dual link based radio frequency fingerprinting for wearable devices. 40th Conf. Local Comput Networks IEEE 1: 329-37.

Sanchez del Rio J, Moctezuma D, Conde C, Martin de Diego I, Cabello E (2016). Automated border control e-gates and facial recognition systems. Comput Secur 62:49-72.

Saxena A, Sharma S, Chaurasiya V (2015). Neural network based human age-group estimation in curvelet domain. Procedia Comput. Sci 54:781-9. 
Schroff F, Kalenichenko D, Philbin J (2015). FaceNet: A Unified Embedding for Face Recognition and Clustering 1:815-23.

Sharma N, Singh DS (2012). A Survey on Various Feature Extraction Techniques and Face Recognition Methodologies using Neural Networks. Int J Adv Res Comput Sci 3:1-4.

Sinitsyna KA (2015). Principles and methods of implementtation of distributed systems based on neural processors. Modern Engineering and Technologies 5:14-8.

Sun Y, Chen Y, Wang X, Tang X (2014). Deep learning face representation by joint identification-verification. Adv Neural Inf 1:1988-96.

Takagi T, Sugeno M (1985). Fuzzy identification of systems and its applications to modeling and control. IEEE Trans Syst Man 1:116-32.
Turk M, Pentland A (1991). Eigenfaces for Recognition. J. Cogn. Neurosci 3:71-86.

Vasenkov D (2007). Learning Methods for Artificial Neural Networks. Computer Tools in Education 1:20-9.

Vinay A, Shekhar V, Rituparna J, Aggrawal T (2015). Cloud based big data analytics framework for face recognition in social networks using machine learning. Procedia Comput 50:623-30.

Watanabe Y, Fujita T (2013). Toward Introduction of Immunity-based Model to Continuous Behavior-based User Authentication on Smart Phone. Procedia Comput Sci 22:1319-27.

Xue J, Su X, Zhang Q (2015). High-speed 3D face measurement based on color speckle projection, Int Conf Exp Mech 8:93022Y-93022Y. 